\title{
SPONTANEOUS PERFORATION OF A MALIGNIFIED CORROSIVE STRICTURE OF THE ESOPHAGUS AND SUBSEQUENT PERFORATION OF A GIANT DUODENAL STRESS ULCUS
}

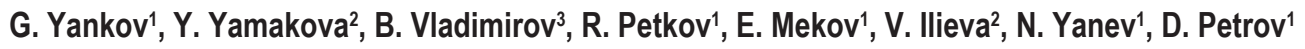 \\ ${ }^{1}$ Department of Respiratory Diseases, Medical Faculty, Medical University - Sofia, Bulgaria \\ ${ }^{2}$ Department of Anesthesiology and Intensive Care, Medical Faculty, Medical University - Sofia, Bulgaria \\ ${ }^{3}$ Department of Gastroenterology, Medical Faculty, Medical University - Sofia, Bulgaria
}

\begin{abstract}
Surgical interventions for corrosive stricture of the esophagus are extremely difficult and technically challenging. In this manuscript, we present a patient with esophagectomy due to perforation of a corrosive stricture of the esophagus that underwent malignant transformation and subsequent perforation of a giant duodenal stress ulcus, which occurred 12 days after the intervention. We performed a total esophagectomy, pharyngo- and gastrostomy, suture of the duodenal perforation but the postoperative period was challenging and despite our efforts, the patient died on the 50th postoperative day due to respiratory and renal failure.
\end{abstract}

Key words: esophageal perforation, corrosive stricture, duodenal perforation, esophageal resection

Corresponding author: MHATPD „Sveta Sofia“, 1431, 19, „Acad. Ivan Geshov" blvd, Sofia, Bulgaria. tel. +359 888320 476, e-mail: evgeni.mekov@gmail.com

\section{CASE REPORT}

W e present the case of a 47-year-old male, who was admitted by emergency in our department of thoracic surgery with complaints of right-sided chest pain, fever up to 39 degrees, malaise, fatigue, and difficulty swallowing. Nineteen years ago he had a corrosive chemical burn of the esophagus with potassium hydroxide $(\mathrm{KOH})$, which he ingested unintentionally. At this time, he had undergone three bougies dilations. He had difficulty swallowing, ate often and in small portions and refused any surgical interventions. The rest of his medical history is remarkable only for pyelonephritis of the right kidney due to calculosis.

On admission the patient was in impaired overall condition with the clinical features of septic shock: pale skin, fever up to 39 degrees, tachycardia, hypotension $(90 / 60 \mathrm{~mm} \mathrm{Hg}$ ) and almost absent breath sounds on the right. There were no clinical signs of an acute surgical abdomen. On the chest $X$-ray, there was a diverticulum-like shadow on the right side of the trachea and oval cyst-like lesion of the lower part of the right lung (Fig. 1).

We performed a chest CT which revealed a rightsided esophageal diverticulum with a hydro-air level within (Fig. 2). Distally to the diverticulum, there was an uneven line of contrast medium that communicates with a thick-sided cavity in the right hemithorax. There were perifocal inflammatory lung infiltrates. In the upper mediastinum, there were air and liquid collections around the diverticulum. The described findings correspond to a fistula between the esophagus and the right hemithorax. 

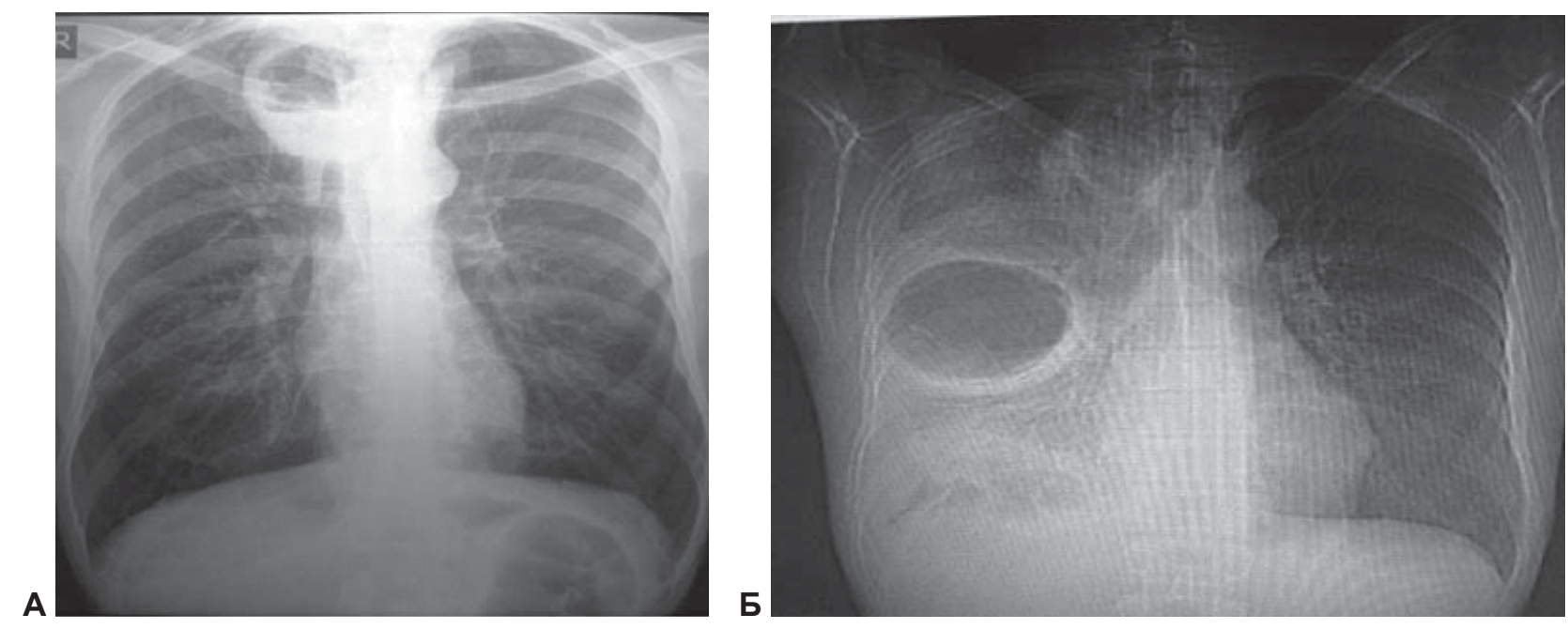

Fig. 1. Chest X-ray. A. One week before perforation; B. Several hours after perforation

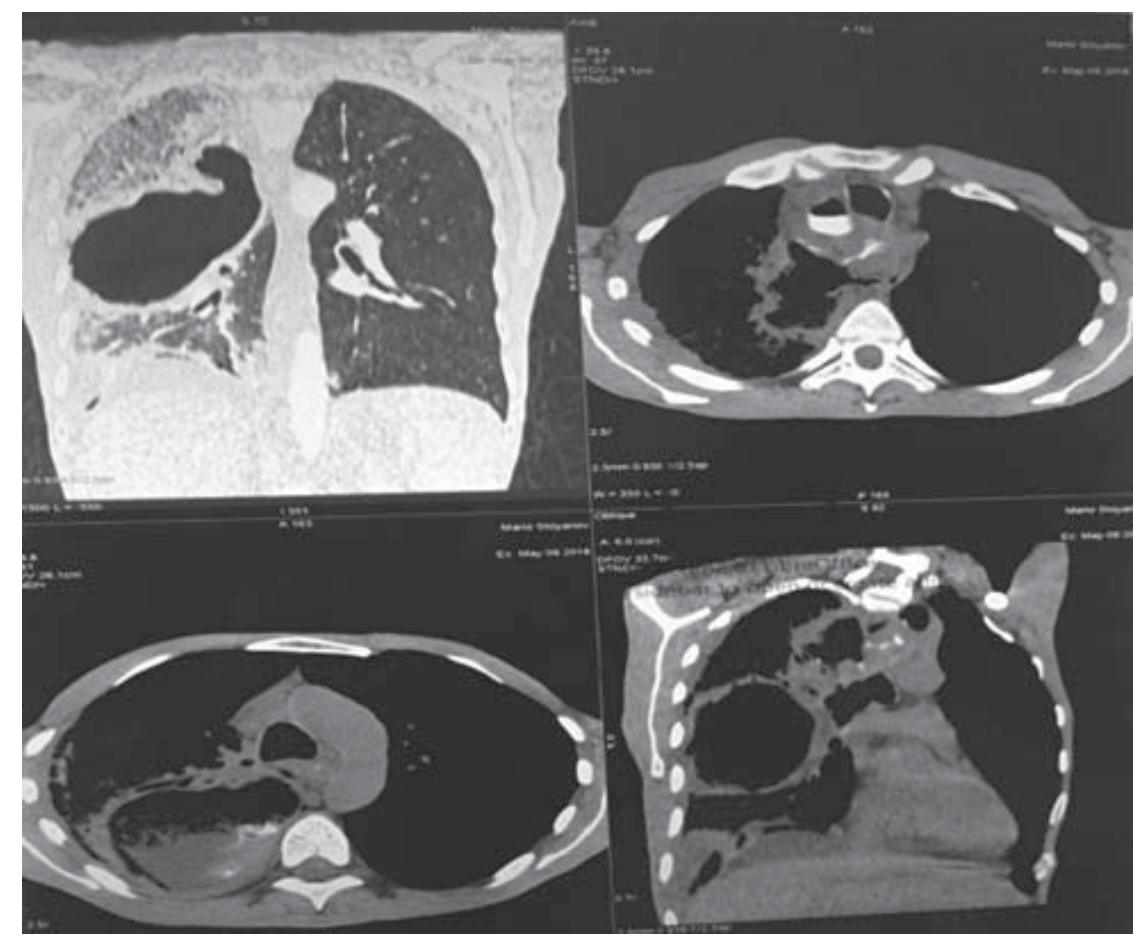

Fig. 2. Chest CT with contrast medium

The patient underwent emergency surgical intervention. During one-lung ventilation, a right thoracotomy was performed. The visceral and parietal pleura were thickened with fibrin depositions and loculations due to purulent empyema. About $1000 \mathrm{ml}$ pus was evacuated. Debridement and decortications of the right lung were performed.

The esophagus was thickened and sclerotic on its entire length. A perforation with dimensions $1 / 1 \mathrm{~cm}$ was found on the proximal part of the thoracic esophagus (Fig. 3). In this area, the esophagus was with a macroscopic appearance of a malignant lesion. There were also massive adhesions due to trans- mural panesophagitis and periesophagitis. Azygos vein was transected and ligated. The esophagus was sharply and bluntly dissected from the thoracic aorta and the aortic arch to the left mainstem bronchus and the trachea to the diaphragmatic hiatus and after that was transected by means of a stapler. In the upper mediastinum, there was an acute fibrinopurulent mediastinitis, while in the posterior mediastinum there was a fibrous proliferative inflammatory process with sclerosing fibrosis.

We performed systematic lymph node dissection of macroscopically inflammatory altered subcarinal and paraesophageal lymph nodes. In the posterior 
mediastinum around the distal trachea, chialorrhea appeared and the ligation of ductus thoracicus was carried out.

By means of left-sided cervicotomy, debridement of a massive adhesion around the sclerotic cervical esophagus was performed. A total esophagectomy and pharyngostomy were performed. At the end of the procedure, a longitudinal transrectal mini-laparotomy and gastrostomy were carried out.

The specimen is shown on Fig 4. The histological result showed a highly-differentiated squamous cell carcinoma with transmural infiltration of the esophageal wall up to the adventitia - G1, pT3N0M0.

The patient was reintubated with a single-lumen endotracheal tube and remained on pressure-controlled positive pressure ventilation with a fraction of inspired oxygen of $50 \%$, tidal volume of $6 \mathrm{ml} / \mathrm{kg}$ and respiratory rate of $18 / \mathrm{min}$. Because of expected long-term mechanical ventilation, an early tracheostomy was performed. The hypovolemia was corrected and antibiotic therapy was started with cefoperazone/sulbactam and metronidazole and stress ulcus prophylaxis with omeprazole $40 \mathrm{mg} /$ day.

On the seventh postoperative day, a left pleural effusion appeared and pleural drainage was inserted.

On the twelfth postoperative day, clinical signs of acute surgical abdomen appeared. An emergency laparotomy was performed. $1000 \mathrm{ml}$ of turbid exudate was evacuated from the peritoneal and retroperitoneal cavities. We found a giant perforated stress ulcus on the anterior duodenal wall (Fig. 5). The duodenum was mobilized with Kocher maneuver, the ulcus was sutured and the suture line was buttressed with omentum and the thickened wall of the gallbladder. The gastrostomy was retained.
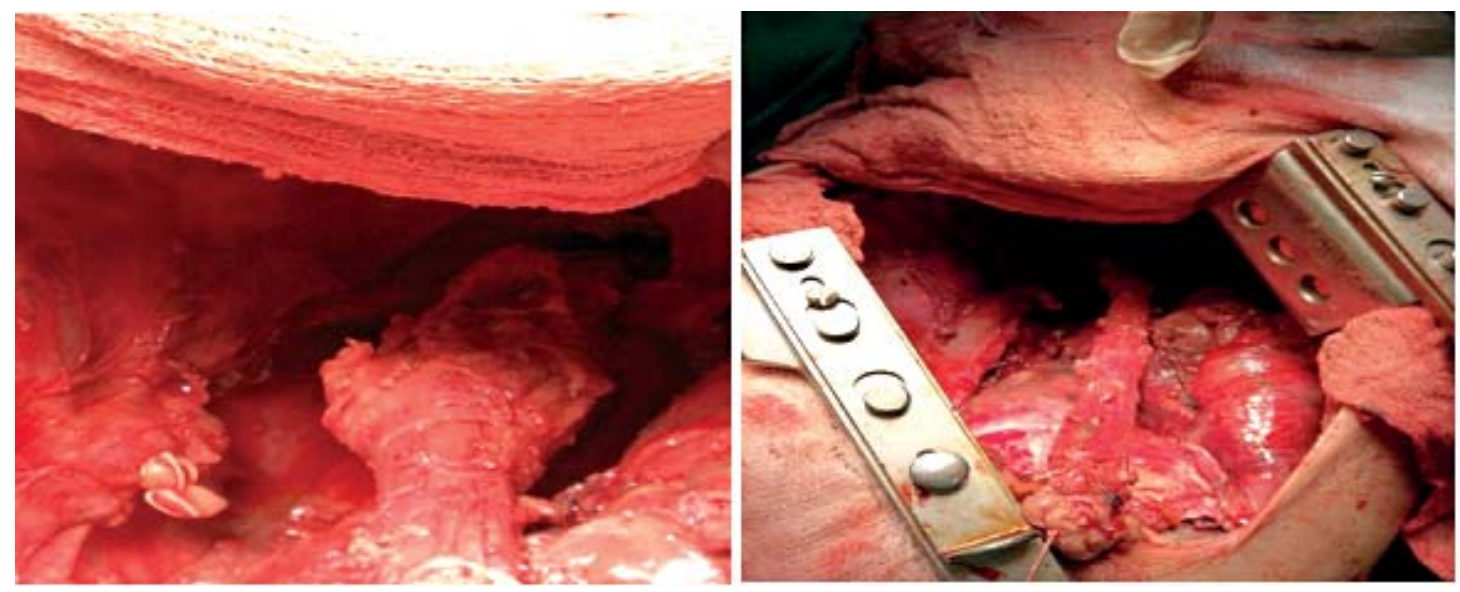

Fig. 3. Intraoperative view of the mobilized esophagus
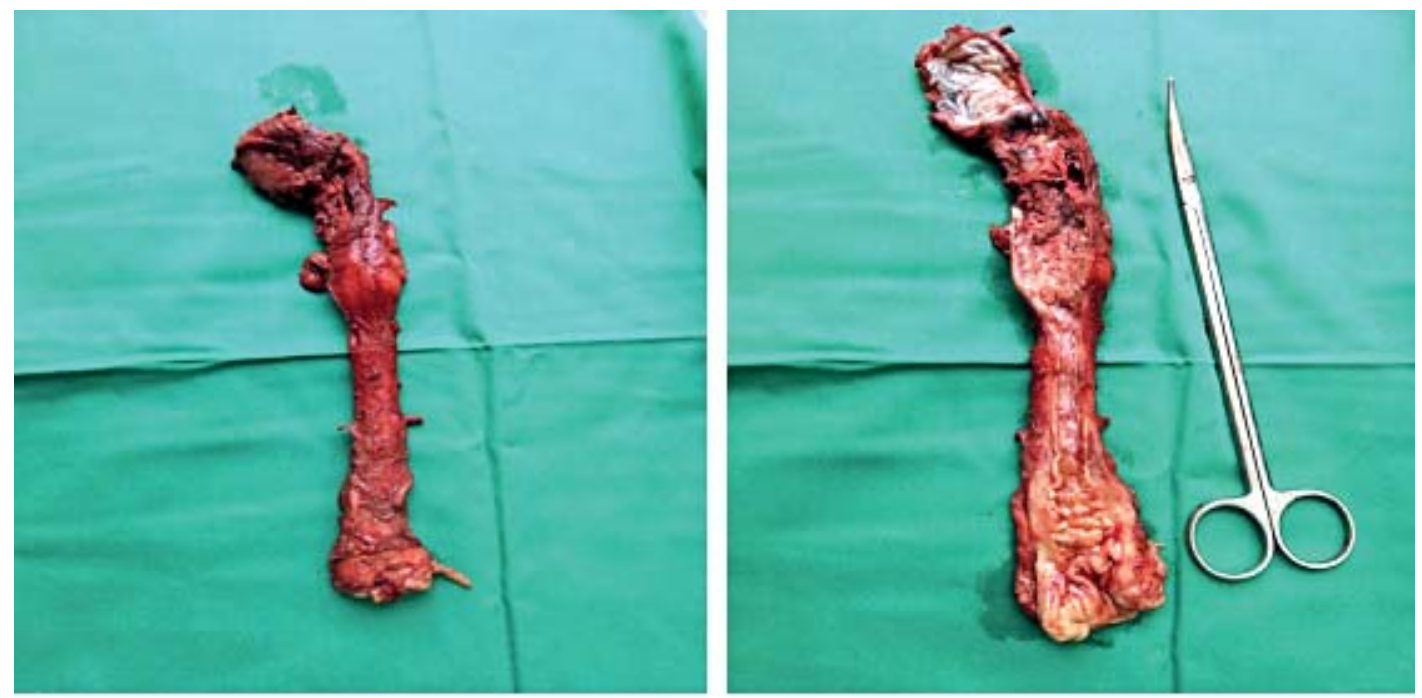

Fig. 4. Pathological specimen of the esophagus 


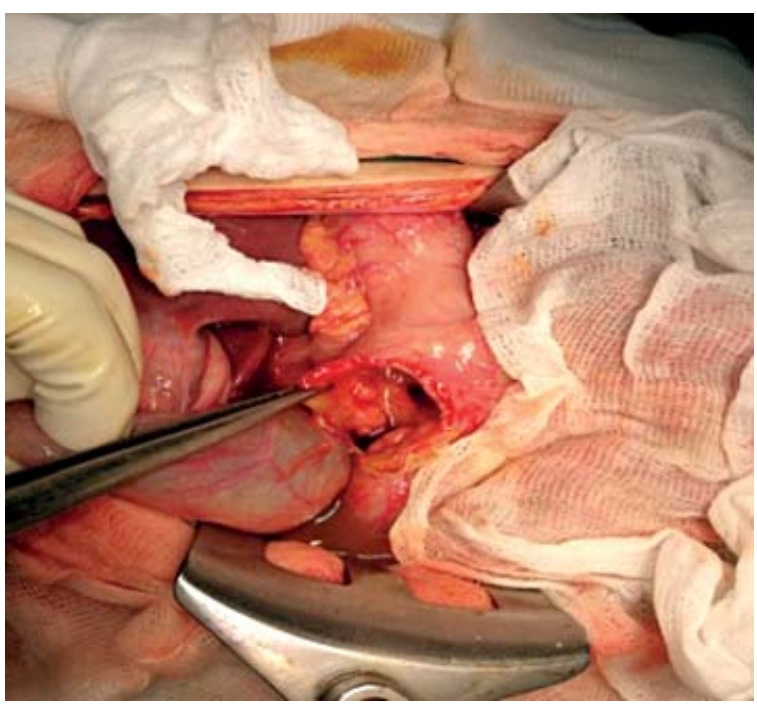

Fig. 5. Giant perforative duodenal ulcus

On the twentieth postoperative day hemorrhagic shock due to massive melena was noted and the hemoglobin decreased to $70 \mathrm{~g} / \mathrm{l}$. The condition was controlled with a blood transfusion, fresh frozen plasma, hemostatic drugs, and proton-pump inhibitors. The omeprazole dose was increased to $80 \mathrm{mg} /$ day, therapy with an $\mathrm{H} 2-$ blocker was initiated, as well as acetamsylate, vitamin $\mathrm{K}$ and calcium gluconate.

Afterwards, the patient developed ventilator-associated pneumonia with ARDS and remained on volumecontrolled mechanical ventilation. The microbiology showed a multiresistant Klebsiella pneumoniae strain (sensitive only to colistin and intermediately sensitive to gentamycin) from the pleural effusion and bronchoalveolar lavage. Pseudomonas aeruginosa was isolated from the tracheal aspirate.

On the forty-eighth postoperative day, the patient developed acute renal failure with oliguria (urine output $100 \mathrm{ml} /$ day) and nitrogen retention (creatinine up to $250 \mu \mathrm{mol} / \mathrm{l}$ ) and was treated with furanthril $320 \mathrm{mg} /$ day. Unfortunately, on the fiftieth postoperative day, the patient died.

\section{DISCUSSION}

The incidence of late malignant transformation of corrosive esophageal strictures is around $13 \%$ and it occurs only with chemical burns, caused by bases and never by acids [1, 2]. Therefore, Kim et al. [2], recommend performing a total esophagectomy in all cases, especially in young patients with long life expectancy. The optimal timing for surgery in corrosive esophageal strictures is still a subject of dispute [3]. In cases of perforation, it is performed in an emergency setting.

Esophageal perforations are associated with high morbidity and mortality [4]. Major prognostic factors are cause and place of the perforation, presence of underlying esophageal pathology, late diagnosis, and treatment method [5-7]. The management of thoracic esophageal perforations is always more challenging than that of cervical or abdominal perforations because of the severe systematic complications, caused by pleural and mediastinal contamination [8]. In our case, early surgical intervention for corrosive stricture was not performed because the patient denied it. Therefore, we were forced to operate in an emergency setting in the extremely difficult circumstances of perforation, mediastinitis, right-sided pleural empyema, impaired overall condition, and severe malnutrition.

A series of postoperative complications followed the initial intervention. After the esophagectomy, the patient could not have been extubated on the operating table and he was put on mechanical ventilation. On the twelfth postoperative day, perforation of a duodenal stress ulcus complicated the condition further but was resolved. The following hemorrhagic shock on the twentieth postoperative day was also managed successfully. The final complication, leading to the fatal outcome was the development of ARDS on the thirty-second postoperative day and acute renal failure. The negative prognostic factors for this unfavorable outcome could be attributed to malnutrition, low serum albumin, sepsis, polyresistant bacterial strains, massive blood transfusions, late hospitalization (1 week after the initial symptoms of mediastinitis) and ventilator-associated pneumonia.

Disclosure Summary: The authors have nothing to disclose.

\section{REFERENCES}

1. Davids PH, Bartelsman JFB, Tilanus HW, van Lanschot JJ. Consequences of caustic damage of the esophagus. Ned Tijdschr Geneeskd 2001;145:2105-8.

2. Kim YT, Sung SW, Kim JH. Is it necessary to resect the diseased esophagus in performing reconstruction for corrosive esophageal stricture? European Journal of Cardio-Thoracic Surgery 2001;20(1):1-6.

3. Han Y, Cheng QS, Li XF, Wang XP. Surgical management of esophageal strictures after caustic burns: A 30 years of experience. World J Gastroenterology 2004;10(9):2846-9.

4. Kaman L, Iqbal J, Kundil B, Kochhar R. Management of Esophageal Perforation in Adults. Gastroenterology Res 2010;3(6):235-44.

5. Altorjay A, Kiss J, Voros A, Sziranyi E. The role of esophagectomy in the management of esophageal perforations. Ann Thorac Surg 1998;65(5):1433-6.

6. Attar S, Hankins JR, Suter CM et al. Esophageal perforation: a therapeutic challenge. Ann Thorac Surg 1990;50(1):45-9.

7. Gupta NM, Kaman L. Personal management of 57 consecutive patients with esophageal perforation. Am J Surg 2004;187(1):58-63.

8. Brinster CJ, Singhai $S$, Lee $L$ et al. Evolving options in the management of esophageal perforation. Ann Thorac Surg 2004;77(4):1475-83. 